\title{
Realization of Optical Logic Gates Using the Thermal Lens Effect
}

\author{
ACHAMMA KURIAN*, K. P. UNNIKRISHNAN, THOMAS LEE S., \\ V. P. N. NAMPOORI ${ }^{\dagger}$ and C. P. G. VALLABHAN \\ International School of Photonics, Cochin University of Science \& Technology, \\ Cochin 682022, India
}

(Received 7 March 2002)

\begin{abstract}
A sensitive method based on the principle of photothermal phenomena to realize optical logic gates is presented. A dual beam thermal lens method using low power $\mathrm{cw}$ lasers in a dye-doped polymer can be very effectively used as an alternate technique to perform the logical function such as NAND, AND and OR.
\end{abstract}

Key words: Photothermal phenomena; Thermal lens; Optical logic gates

\section{INTRODUCTION}

Logic operations are nonlinear and hence obviously nonlinear optics play a vital role in the realization of a digital optical computer. Availability of coherent optical sources along with the realization of various nonlinear optical phenomena has made it possible to implement optics based computing and memory elements. It is now possible to control a light beam with another using nonlinear optical effect. This has helped in developing various types of optical logic gates based on optical phase conjugation [1-4], optical bistability [5-7], optical interference [8], hybrid self-electrooptic effects $[9,10]$, image subtraction [11] etc.

Another way of realizing optical logic gates is by using a well-known photothermal phenomenon called the thermal lens effect (TL). Eversince the discovery of the photothermal lensing effect by Gordon et al. [12] this technique of monitoring nonradiative relaxation in excited molecule has

\footnotetext{
* Corresponding author. Permanent address: Catholicate College, Pathanamthitta 689645, India. E-mail: achammakurian@rediffmail.com

$\dagger$ E-mail: vpnnampoori@cusat.ac.in
} 
been refined by various researchers for the study of various phenomena related to light-matter interactions [13-16]. When a medium is illuminated with a Gaussian laser beam, some of the energy is absorbed by the molecules in the ground state and they are excited to higher energy states. The excess energy attained by the molecule can be dissipated in many ways. The non-radiative decay process causes the heating of the sample, which creates a refractive index gradient in the medium and the resulting refractive index profile produces a thermal lens. This negative lens causes beam divergence and the signal is detected as a time dependent decrease in power at the center of the beam.

Above a certain threshold laser intensity, the refractive index gradient in the medium becomes so large that the probe beam gets diverged to form a bottle like shape with central dark region surrounded by a bright region. Such modification of the probe beam shape can be used to implement optical logic gates due to the existence of threshold like phenomenon in the TL effect. Changes in the optical intensity at the center of the probe beam at far field due to the thermal lens effect can be used as the thermal lens signal. The exact level of the threshold determines which Boolean function appears at the output. One can modify the scheme of data detection to realize various types of logic gates.

\section{MATERIALS AND METHOD}

The experimental setup for the dual beam thermal lens method for realizing logic gates is shown in Figure 1. Laser radiation at $532 \mathrm{~nm}$ wavelength from a Diode Pumped Nd: $\mathrm{YVO}_{4}$ laser (Uniphase BWT-50) is split in to two beams $I_{1}$ and $I_{2}$. These pump beams are then spatially overlapped in the medium to generate a thermal lens effect. A low power $(1 \mathrm{mw})$ intensity stabilized He-Ne laser source of wavelength $632.8 \mathrm{~nm}$ is used as the probe beam. The sample in the form of a disc (thickness $3 \mathrm{~mm}$ and $1 \mathrm{~cm}$ diameter) is kept in the pump beam path. The probe beam is made to pass collinearly through the sample using a dichroic mirror. A filter is placed in the path of the emergent beams, which allows only the $632.8 \mathrm{~nm}$ wavelengths to reach the photodiode.

The first observation of stimulated emission from polymer matrices doped with organic dyes were reported as early as 1967 and 1968 by Soffer and McFarland [17] and Peterson and Snavely [18], respectively. Since then a number of solid organic and inorganic polymeric matrices have been described $[19,20]$. However, the use of poly(methyl methacrylate) (PMMA) host presents additional advantages as these materials show a 


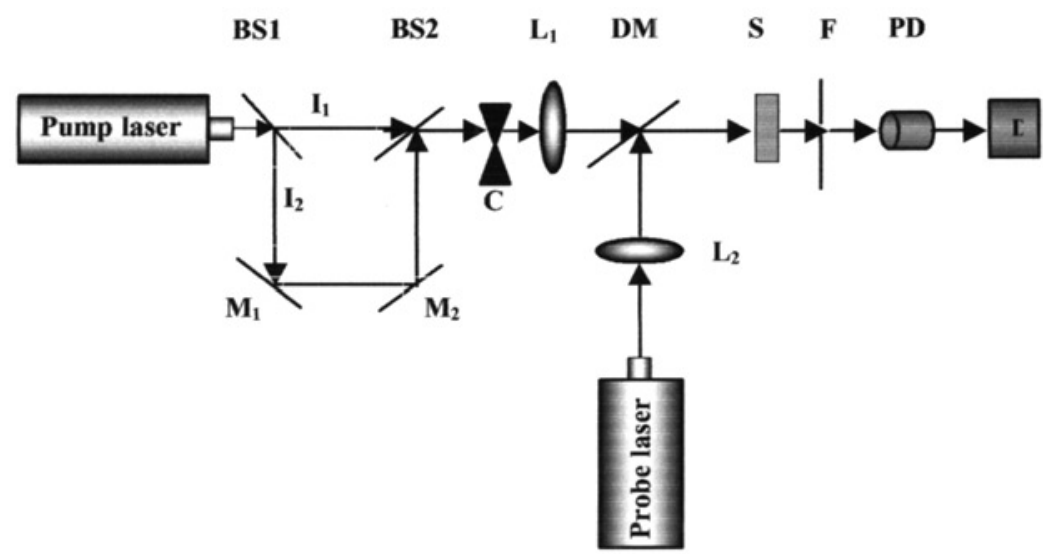

FIGURE 1 Schematic diagram of the experimental set up for realizing logic gates. BS1, BS2 - Beam Splitters, $\mathbf{C}$ - Chopper, $\mathbf{L}_{\mathbf{1}}, \mathbf{L}_{\mathbf{2}}$ - Lens, DM - Dichroic Mirror, $\mathbf{S}$ - Sample, $\mathbf{F}$ - Filter, PD - Photodiode, D - Detector.

much better compatibility with organic laser dyes and are amenable to inexpensive fabrication techniques which combined with their light weight would facilitate miniaturization and the design of integrated optical systems [21-24]. Optical media used for our present study are chemically stabilized Rhodamine 6G doped PMMA, due to its best optical transparency and resistance to laser damage [25-27].

Methyl methacrylate (Merck) is washed three times with 2 vol\% aqueous sodium hydroxide to remove the inhibitor and then twice with distilled water. An accurately weighed amount of Rh 6G (Exciton) is dissolved in a mixture of methyl methacrylate (MMA) and ethanol taken in the ratio 4:1 to give a concentration of $4 \times 10^{-3} \mathrm{moll}^{-1}$. Then $1.5 \mathrm{gm} / 1$ of benzoyl peroxide, a free-radical initiator, is added to each sample solution. The mixture is taken in glass bottles and is kept in a constant temperature bath maintained at $50{ }^{\circ} \mathrm{C}$. After about 48 hours completely polymerized samples are taken out and kept for one week for drying. Then the samples are cut into pieces having a thickness of $3 \mathrm{~mm}$ and are polished to minimize losses due to scattering.

\section{RESULTS AND DISCUSSION}

A simple implementation of binary logic gates using a nonlinear effect is where the nonlinearity consists of a threshold located at some input level 
$[28,29]$. Threshold like behavior of an aberrated thermal lens effect is shown in Figure 2. In the experiment to realize NAND gate, when the both pump beams $\left(I_{1}\right.$ and $\left.I_{2}\right)$ are absent, the output corresponds to the intensity of the probe beam itself detected by the photodiode which gives the first condition in Table I. If the input of either beam is below the threshold there is a high output (second and third condition of Tab. I). If both inputs are present thermal blooming is so strong that the output of the photodetector is low which is taken as zero. The output-input relations of the TL experiment, using the photodetector output as the signal, satisfy a NAND gate type relation as shown in Table I. Logical 1 is represented by high light intensity while zero is represented by low intensity.

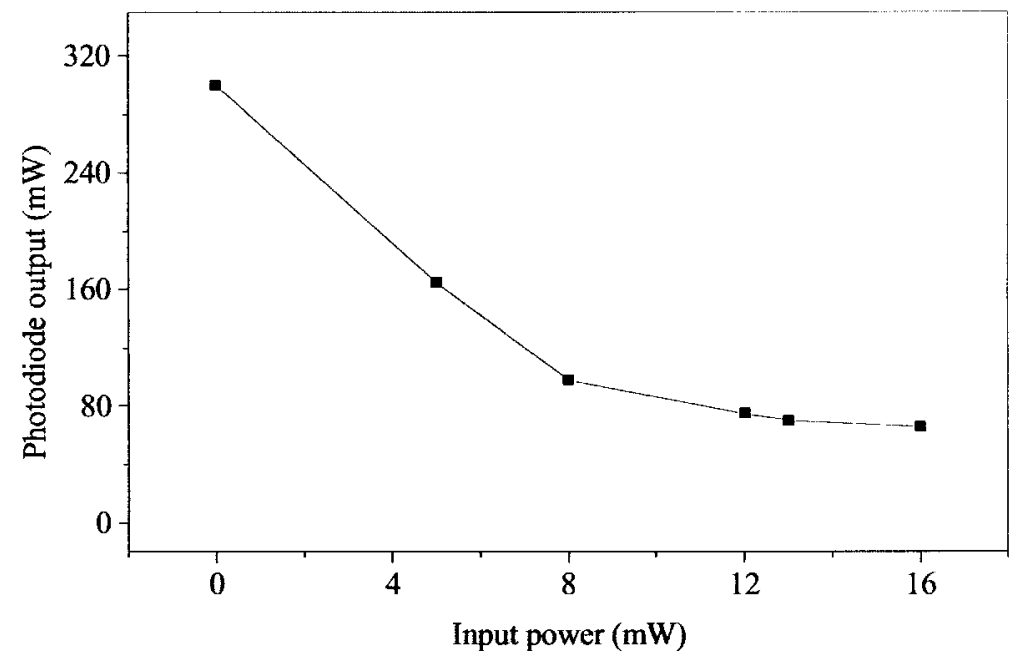

FIGURE 2 Power dependence of the NAND gate (concentration of the sample $\left.4 \times 10^{-3} \mathrm{moll}^{-1}\right)$.

TABLE I NAND Gate.

\begin{tabular}{lll}
\hline$I_{1}$ (input) & $I_{2}$ (input) & $\begin{array}{l}\text { Photodiode } \\
\text { output }\end{array}$ \\
\hline 0 & 0 & 1 \\
0 & 1 & 1 \\
1 & 0 & 1 \\
1 & 1 & 0 \\
\hline
\end{tabular}


In order to implement the AND gate the thermal lens signals for different pump powers are obtained which is shown in Figure 3. If the input is below the threshold, the thermal lens signal is low. From the figure it is clear that when both pump beams are absent the thermal lens signal is zero, which gives the first condition of Table II. If either $I_{1}$ or $I_{2}$ is less than $I_{s}$ (see Fig. 3 ), thermal lens signal is low which can be considered as zero. $I_{1}$ and $I_{2}$ together gives an augmented thermal lens signal which corresponds to the last condition of Table II. Saturation of intensity, $I_{s}$, depends on the concentration of the dye in the matrix. By adjusting the pump powers we can implement the OR gate. When both the inputs are low the TL signal is low. However, if we adjust the inputs such that $I_{1}$ or $I_{2}$ is greater than $I_{s}$ the output is high (Tab. III). Absorbed power must not be so large to introduce

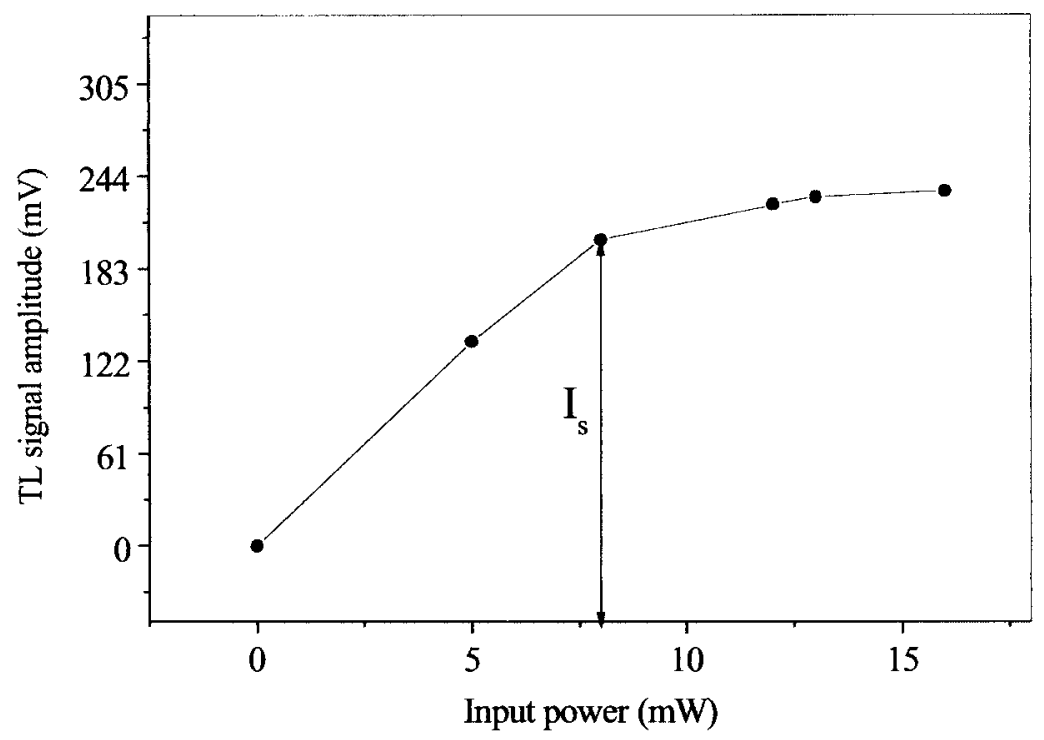

FIGURE 3 Input-output characteristics for AND gate.

TABLE II AND Gate.

\begin{tabular}{ccc}
\hline$I_{1}$ (input) & $I_{2}$ (input) & TL output \\
\hline 0 & 0 & 0 \\
0 & 1 & 0 \\
1 & 0 & 0 \\
1 & 1 & 1 \\
\hline
\end{tabular}


TABLE III OR Gate.

\begin{tabular}{ccc}
\hline$I_{1}$ (input) & $I_{2}$ (input) & TL output \\
\hline 0 & 0 & 0 \\
0 & 1 & 1 \\
1 & 0 & 1 \\
1 & 1 & 1 \\
\hline
\end{tabular}

photodegradation of the dye and aberration of the thermal lens. It is a wellestablished fact that the PMMA matrix does not undergo any change in its chemical, or any other physical properties, at very low laser powers as used here [25-27].

To conclude, the thermal lens technique has been successfully implemented for realizing basic logic gates operated with optical beams of moderate intensity. The method is based on the divergence of the lens formed by the non-radiative decay within the sample due to the pump beam. In contrast to other optical logic gates this method is sensitive as well as it does not require any nonlinear optical materials. We have used thin samples made of Rhodamine 6G doped PMMA, where one beam can control the intensity of a second beam.

\section{Acknowledgements}

The authors are grateful to UGC (Govt. of India) for financial assistance. KPU and TLS are thankful to the CSIR (Govt. of India) for financial help. The authors also acknowledge the financial support from the Netherlands University Federation for International Collaboration (NUFFIC).

\section{References}

[1] Abraham, E., Seaton, C. T. and Smith, S. D. (1983) Scientific American, 2489, 85.

[2] Flruret, J. (1984) Appl. Opt., 23, 1609.

[3] Fainman, Y., Guest, C. C. and Lee, S. H. (1986) Appl. Opt., 25, 1598.

[4] Eichmann, G., Li, Y. and Alfano, R. R. (1987) Appl. Opt., 26, 194.

[5] Smith, P. W. (1980) Opt. Engg., 19, 456.

[6] Smith, P. W. and Tomlinson, W. J. (1981) IEEE Spectrum, 18, 26.

[7] Pepper, D. M. (1982) Opt. Engg., 21, 156.

[8] Wang, L. A., Chang, S. H. and Lin, Y. F. (1998) Opt. Eng., 37, 1011.

[9] Miller, D. A. B. (1982) Laser Focus, 18, 79.

[10] Miller, D. A. B. (1983) Laser Focus, 19, 61.

[11] Ebersole, J. F. (1975) Opt. Eng., 14, 435.

[12] Gordon, J. P., Leite, R. C. C., Moore, R. S., Porto, S. P. S. and Whinnery, J. R. (1965) J. Appl. Phys., 36, 3.

[13] Hue, C. and Whinnery, J. R. (1973) Appl. Opt., 12, 72. 
[14] Snook, R. D. and Lowe, R. D. (1995) Analyst, 120, 2051.

[15] Bindhu, C. V., Harilal, S. S., Kurian, A., Nampoori, V. P. N. and Vallabhan, C. P. G. (1998) J. Nonlinear Opt. Phys. \& Mats., 7, 531.

[16] Kurian, A., Unnikrishnan, K. P., Gopinath, P., Nampoori, V. P. N. and Vallabhan, C. P. G. (2001) J. Nonlinear Opt. Phys. \& Mats., 10, 415.

[17] Soffer, B. H. and McFarland, B. B. (1967) Appl. Phys. Lett., 10, 266.

[18] Peterson, O. G. and Snavely, B. B. (1968) Appl. Phys. Lett., 12, 238.

[19] Suzuki, A., Ishii, T. and Maruyama, Y. (1996) J. Appl. Phys., 80, 131.

[20] Gupta, M., Sharma, V. K., Kapoor, A. and Tripathi, K. N. (1997) J. Opt., 28, 37.

[21] Franke, H. (1984) Appl. Opt., 23, 2729.

[22] Welker, D. J. and Kuzyk, M. G. (1996) Appl. Phys. Lett., 69, 1835.

[23] Sasaki, K. and Nagamura, T. (1998) J. Appl. Phys., 83, 2894.

[24] Magda A.El-Shahawy (2000) Polymer Testing, 19, 821-829.

[25] Costela, A., Garcia-Moreno, I., Figuera, J. M., Amat-Guerri, F. and Sastre, R. (1998) Laser Chem., 18, 63-84.

[26] Arbeloa, F. L., Lopez Arbeloa, T., Lopez Arbeloa, I., Costela, A., Garcia-Moreno, I., Figuera, J. M., Amat-Guerri, F. and Sastre, R. (1997) Appl. Phys., B 64, 651.

[27] Nibu, A. G., Aneeshkumar, B., Radhakrishnan, P. and Vallabhan, C. P. G. (1999) J. Phys. D: Appl. Phys., 32, 1745.

[28] Abdeldayem, H., Frazier, D. O., Paley, M. S. and Witherow, W. K. Recent Advances in Photonic Devices for Optical Computing, NASA Marshall Space Flight Center, Space Sciences Laboratory, Huntsville, al 35812

[29] Sawchuk, A. A. and Strand, T. C. (1984) Proc. IEEE, 72, 758. 